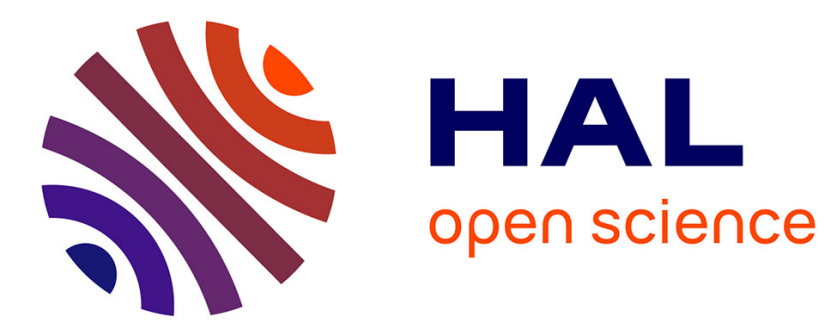

\title{
Light-induced High-spin State in ZnO Nanoparticles
}

Adrien Savoyant, M Rollo, M. Texier, R. Adam, S. Bernardini, O. Pilone, O. Margeat, O Nur, M Willander, S. Bertaina

\section{To cite this version:}

Adrien Savoyant, M Rollo, M. Texier, R. Adam, S. Bernardini, et al.. Light-induced High-spin State in ZnO Nanoparticles. Nanotechnology, 2019, 10.1088/1361-6528/ab57f1 . hal-02321699

\section{HAL Id: hal-02321699 \\ https://hal.science/hal-02321699}

Submitted on 21 Oct 2019

HAL is a multi-disciplinary open access archive for the deposit and dissemination of scientific research documents, whether they are published or not. The documents may come from teaching and research institutions in France or abroad, or from public or private research centers.
L'archive ouverte pluridisciplinaire HAL, est destinée au dépôt et à la diffusion de documents scientifiques de niveau recherche, publiés ou non, émanant des établissements d'enseignement et de recherche français ou étrangers, des laboratoires publics ou privés. 


\title{
Light-induced High-spin State in ZnO Nanoparticles
}

\author{
A. Savoyant, ${ }^{1}$ M. Rollo, ${ }^{1}$ M. Texier,${ }^{1}$ R. E. Adam,${ }^{2}$ S. Bernardini, ${ }^{1}$ \\ O. Pilone, ${ }^{1}$ O. Margeat ${ }^{3}$ O. Nur, ${ }^{2}$ M. Willander, ${ }^{2}$ and S. Bertaina ${ }^{1}$ \\ ${ }^{1}$ Aix Marseille Univ, Université de Toulon, CNRS, IM2NP, Marseille, France \\ ${ }^{2}$ Department of Science and Technology (ITN), \\ Linköping University, SE-601 74 Norrköping, Sweden \\ ${ }^{3}$ Aix Marseille Univ, CNRS, CINAM, Marseille, France
}

\begin{abstract}
The effects of white-light irradiation on $\sim 20 \mathrm{~nm}$ diameter $\mathrm{ZnO}$ nanoparticles are investigated by means of electron paramagnetic resonance, near liquid-nitrogen and liquid-helium temperatures. Under dark conditions, usual core- and surface-defects are detected, respectively at $g=1.960$ and $g=2.003$. Under white-light illumination, the core-defect signal intensity is strongly increased, which is to be correlated to the light-induced conductivity's augmentation. Beside, a four-lines structure appears, with the same gravity center as that of the surface defects. Simulations and intensity power-dependence measurements show that this four-line-structure is very likely to arise from a localized high spin $S=2$, induced by light irradiation, and subjected to a weak axial anisotropy. At $85 \mathrm{~K}$, this high-spin state can last several hours after the light-irradiation removal, probably due to highly spin-forbidden recombination process. The possible excited resonant complexes at the origin of this signal are discussed. Other light-induced $S=1 / 2$-like centers are detected as well, which depends on the nanoparticles growth conditions.
\end{abstract}

PACS numbers: 32.30.Dx, 32.80.Fb, 75.75.-c, 76.30.-v

\section{INTRODUCTION}

Zinc Oxide $(\mathrm{ZnO})$ is an extensively studied metal oxide semiconductor, due to its remarkable physical properties ${ }^{1}$. It is a n-type wurtzite semiconductor with a direct bandgap of about $3.37 \mathrm{eV}$ at room temperature and relatively large exciton binding energy of $60 \mathrm{meV}$. Naturally present in abundance, non toxic and of low cost, $\mathrm{ZnO}$ material is widely used in electronic and optoelectronic due to its high electron mobility ${ }^{2}$, photoelectric response ${ }^{3}$, excellent chemical and thermal stability ${ }^{4,5}$, piezoelectric applications ${ }^{6}$, gas sensors ${ }^{7-9}$, light emitting diode ${ }^{10,11}$, and so on.

From the physical point of view, one of the most striking characteristic of $\mathrm{ZnO}$ is its strong interaction with light, resulting in photo-induced effets, such as photo-conductivity ${ }^{12,13}$, photocatalysis $^{14-17}$ and photo-luminescence ${ }^{18,19}$. All of these effects are mainly driven by excitonic and point-defects related properties, as well as coupling of both ${ }^{20-24}$. In nanosized $\mathrm{ZnO}$ systems, these photo-induced effects can be affected by the important surface-to-volume ratio, and/or by the presence of specific point-defects acting for example as new recombination or charge donor/acceptor centers. The study of these sizeinduced defects is thus a matter of great interest for tailoring efficient nanostructured $\mathrm{ZnO-based}$ devices.

Electron paramagnetic resonance (EPR) is a tool of choice to study point defects. This technique has been used for more than fifty years for characterizing intrinsic and extrinsic param- agnetic defects in bulk $\mathrm{ZnO}^{25-31}$. Regarding $\mathrm{ZnO}$ nanostructures, EPR studies have shown that two major kinds of intrinsic paramagnetic defects are almost always present within, resonating at $g=1.960$ and $g=2.003$, and arising respectively from core- and shell-defects ${ }^{32-34}$. However, the exact nature of these defects is still under debate. A mean of gaining insight into the EPR lines origin is to introduce an additional excitation during the experiment, such as an external electric field or an optical light beam. Indeed, the idea of applying a light irradiation during an EPR experiment goes back to the very early stage of its application on semiconductor materials, where light is likely to ionize point defects into intraband-gap states ${ }^{35,36}$. Regarding $\mathrm{ZnO}$ materials, most of these photo-EPR experiments have been performed on bulk single crystal ${ }^{37-39}$, showing in particular the apparition of some angle-dependent lines, attributed to excited oxygen and zinc vacancies $^{40}$. For $\mathrm{ZnO}$ nanostructures, the main result of such an irradiation is to greatly increase the core-defect $(g=1.960)$ intensity and, to a lesser extent, that of the shell-defect $(g=2.003)^{41}$. However, such photo-EPR studies have not been exhaustively performed onto $\mathrm{ZnO}$ nanoparticles (NPs), in particular regarding their size, growth method and morphology, all characteristics known to greatly influence the population of defects.

In this work, we report on photo-EPR experiment performed on two types of ZnO NPs, near liquid-helium and liquid-nitrogen temperatures. The main new feature observed here is a four-line structure whose gravity center is that of the shell-defect (SD), $g=2.003$, reversibly induced by white light irradiation. Microwave (MW) 
power-dependence study near liquid and helium temperature reveals sub-structures of this four-line group, as well as other photo-induced $S=1 / 2$-like lines, not apparently linked together. This fourlines group appears to be highly saturated around $10 \mathrm{~K}$ and still partially saturated at $85 \mathrm{~K}$. Simulations of the unsaturated spectrum strongly support a $S=2$ effective-spin origin, subjected to a weak axial anisotropy. This effective high-spin state is likely to arise from particular excitonic complex, involving one or more point defects.

In this paper, after explaining the experimental details of materials and method (Sec. II), we present the raw EPR results under dark (Sec. III A) and illuminated (Sec. IIIB) conditions, followed by spectra simulation based on a $S=2$ spin state (Sec. III C). Finally, a qualitative discussion about the origin of this high-spin state is given in the last section (Sec. IV).

\section{EXPERIMENTAL}

The reported EPR measurements were performed on two kinds of hydrothermally grown ZnO NPs, NP1 and NP2, with slightly different preparation methods, but important morphological difference.

NP1 were prepared as published elsewhere ${ }^{42}$. In a typical experiment, a solution of $0.4415 \mathrm{~g}$ of potassium hydroxide $\mathrm{KOH}$ (Aldrich Chemicals 99.99\%) in $23 \mathrm{~mL}$ of anhydrous methanol (Alfa Aesar) was added dropwise to a solution of $0.8202 \mathrm{~g}$ of zinc acetate (Aldrich Chemicals 99.99\%) in $42 \mathrm{~mL}$ of anhydrous methanol and $0.25 \mathrm{~mL}$ of distilled water at $60^{\circ} \mathrm{C}$ under magnetic stirring. After $2 \mathrm{~h} 30 \mathrm{~min}$ at this temperature, the mixture was centrifuged (7800 rpm, $30 \mathrm{~min}$ ) and the supernatant discarded. The product was washed with methanol and centrifuged, then finally dried under vacuum to obtain the $\mathrm{ZnO}$ nanoparticles as powder.

For NP2 fabrication ${ }^{43}$, Zinc acetate dehydrate $(0.1 \mathrm{M})$ and sodium hydroxide $(0.2 \mathrm{M})$ solutions were prepared separately and magnetically stirred overnight at room temperature. Then, the two solutions were mixed into one beaker and magnetically stirred at $750 \mathrm{rpm}$ for 2 hours at temperature of $60^{\circ} \mathrm{C}$. The precipitated white milky solution was separated by centrifugation at $4500 \mathrm{rpm}$ for 2 minutes. Afterward, the precipitation product was washed several times with deionized water and once time with acetone. Finally, the ZnO NPs in the form of powder was obtained by drying the precipitation product into a laboratory oven at $75^{\circ} \mathrm{C}$ for 6 hours.

Transmission electron spectroscopy (TEM) characterizations of both samples (see Fig. 1) show that NP1 are almost spherical in shape with typical diameter of $15 \mathrm{~nm}$, while NP2 have spindle-like shape with $400 \mathrm{~nm}$ and $100 \mathrm{~nm}$ in long and short dimensions, respectively. This points to the great versatility for size and shape of $\mathrm{ZnO}$ NPs, and to its great sensitivity to growth parameters. Both NP1 and NP2 NPs are single crystalline. Most of the presented results are obtained on NP1 sample. Results on NP2 sample are briefly presented at the end of Sec. III C, and mainly serve to prove the reproducibility of the observed photo-induced EPR signal in other types of ZnO NPs.

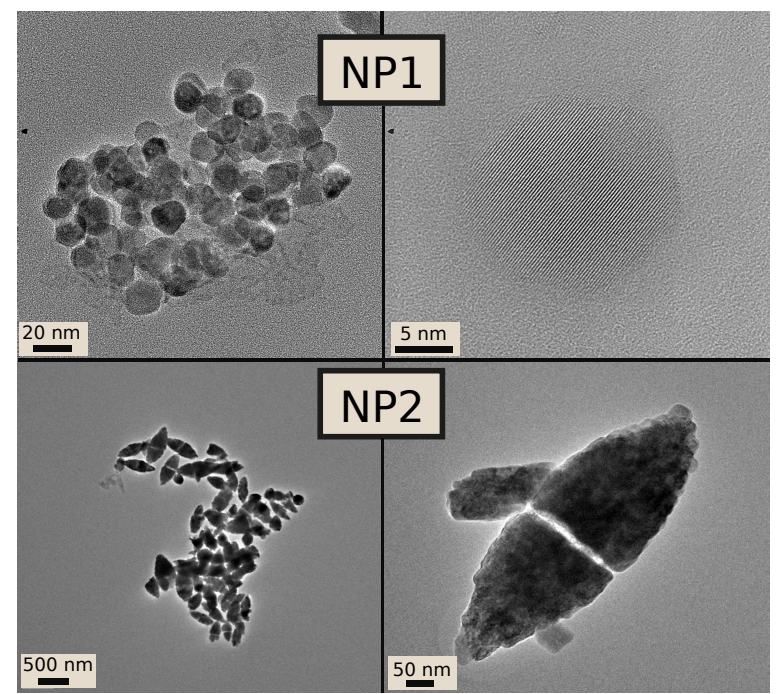

Figure 1: (color online) Transmission electron spectroscopy images of NP1 and NP2 samples, showing differences in size and morphology.

EPR measurements have been performed on a conventional Bruker EMX continuous wave spectrometer operating at X-band $(\nu=9.402 \mathrm{GHz})$ using a standard $\mathrm{TE}_{102}$ mode cavity with optical access. The low $(11 \mathrm{~K})$ and intermediate $(85 \mathrm{~K})$ temperature conditions were obtained by cooling down the samples with liquid helium and nitrogen within a cryostat inserted in the EPR cavity. The microwave (MW) incident power was controlled by an attenuator, allowing power value from 0 to 200 $\mathrm{mW}\left(P_{0}\right)$. Improvement of the signal-to-noise ratio has been achieved by scanning EPR spectra from 4 to 81 times.

White-light irradiation was performed by focusing the photon beam from halogen lamp toward the sample, via the cavity hole. The white-light source has been calibrated so that an optical power of approximatively $80 \mathrm{~mW}$ is reached on the sample. Additional experiment with violet laser mentioned in Sec. IV, was performed with a Radius 405 (nm) laser of $20 \mathrm{~mW}$. 


\section{RESULTS}

\section{A. Light-off EPR spectra}

In figure 2, we present the results of EPR experiments recorded on the NP1 sample at temperatures $T=9 \mathrm{~K}$ and $T=85 \mathrm{~K}$, under dark conditions (light off). Two lines are observed, at $g=1.960$ and $g=2.003$, which have been reported many times in EPR measurement of $\mathrm{ZnO}$ nanostructures ${ }^{32-34}$. They are commonly identified as arising from core- and shell-defect, respectively. The $g=1.96$ signal arises from most of the $\mathrm{ZnO}$ nanostructures ${ }^{44,45}$, regardless of growth method and morphology, but also from bigger microsized crystal as ceramics and bulk single crystal $^{25,37}$. On the contrary, the $g=2.003$ signal only appears in NPs with the smallest diameter $(<50 \mathrm{~nm})$, when surface defects are assumed to play an important role. This is the foundation of the core-shell model (CSM) proposed by Erdem et al., which describe a very stable ZnO NP's core, coated by a shell subjected to interactions with the surroundings ${ }^{32,33}$.

The NP2 sample spectrum (not shown) displays identical core-defect (CD) signal, while SD signal is not detected at none of the studied temperatures. This is consistent with the NPs bigger size in NP2, for which SD signal is not expected to occur.

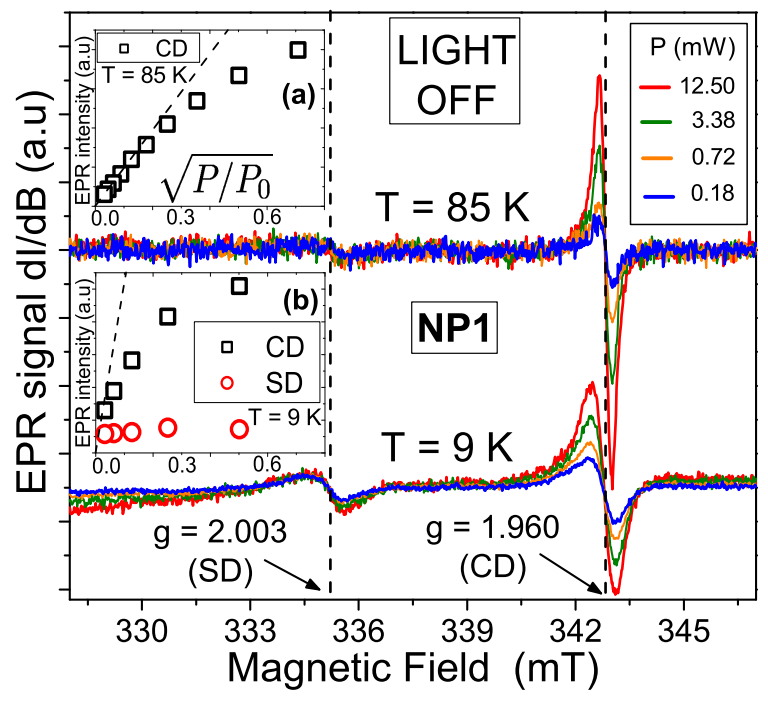

Figure 2: (color online) Dark EPR spectra of $\mathrm{ZnO}$ nanoparticles recorded at $T=9$ and $85 \mathrm{~K}$ on NP1 sample, with different MW powers, showing the shelldefect (SD) and core-defect (CD) lines. Insets (a) and (b) show the saturation curve of $\mathrm{CD}$ at $85 \mathrm{~K}$ and those of $\mathrm{CD}$ and $\mathrm{SD}$ at $9 \mathrm{~K}$, respectively.

From the power-dependence of NP1 EPR lines intensity (insets (a) and (b) of fig. 2), qualitative information on the spin's dynamics is obtained. If not saturated, EPR intensities vary as the squareroot of incident the MW power, which results in straight dashed lines in the insets of figure 2. All departures from these lines indicate a (partially or totally) saturated spin resonance.

It can be seen that the SD signal saturates at weaker power than the CD one, so that we can draw the rough picture that SD has spin-lattice relaxation time significantly greater than that of the CD. This may be understood within the coreshell model of NPs, in which the CD spin must be more strongly coupled/linked to the lattice than the SD spin.

\section{B. Light-on EPR spectra}

When the white-light source is turned on and focalized on samples, two main drastic changes are observed in the EPR spectra.

First, the CD signal's intensity is greatly enhanced with time constant of several minutes at RT, depending on the power light intensity. The intensity reaches a saturation value which also depends on the light-power intensity. This first point is correlated to the observed photoconductivity in $\mathrm{ZnO} \mathrm{NPs}^{30}$. When white-light is turned off, the enhanced CD signal lasts for several hours at $85 \mathrm{~K}$, which is certainly related to the (problematic) persistent photoconductivity observed in the $\mathrm{ZnO}$ material ${ }^{12,13,21}$. The $g=1.960$ signal thus certainly arises from a donor center, located within the NPs core.

The second remarkable change in the EPR spectra is the apparition of a four-line structure (FLS), whose gravity center is that of the SD signal. This is the photo-induced EPR signal studied and discussed in this paper.

Figure 3 shows the result of white-light illumination during an EPR experiment on NP1 sample: an intense four line structure (FLS) appears, centered at the SD $g$-factor (2.003), and whose line are denoted $\mathrm{L}_{1}$ to $\mathrm{L}_{4}$. Remarkably, these new lines have equal spacing $(\Delta=2.25 \mathrm{mT})$ and equal peak-to-peak line width $\left(L_{p p}=0.4 \mathrm{mT}\right)$, pointing to a single origin, possibly related to the SD. Such a relation is not obvious because the $\mathrm{SD}$ g-factor is so close to its free-electron value (2.0023), where every isotropic $1 / 2$ spin resonate. We note that the observed lines shape are clearly not those of a simple EPR line, due to unresolved lines in between and/or to saturation phenomena.

In order to get insight into the relaxation dynamics of these EPR transitions, the temperature is slightly increased (from 9 to $11 \mathrm{~K}$ ) and the MW power is varied from 0.007 to $1.584 \mathrm{~mW}$. As shown in figure 4, three new features are observed. (1), 


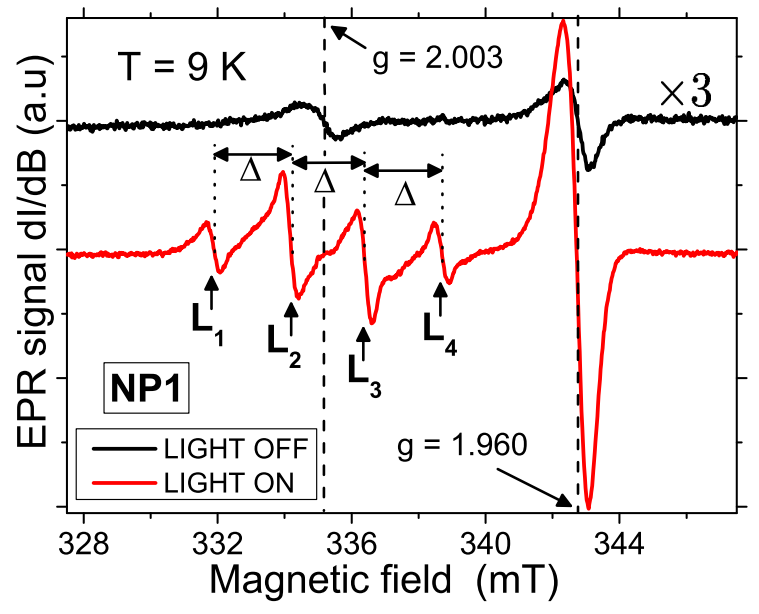

Figure 3: (color online) Dark (up, black) and illuminated (down, red) EPR spectra of ZnO NPs, recorded at $9 \mathrm{~K}$ on NP1 sample. The dark spectrum is magnified by factor $3 . \Delta=2.25 \mathrm{mT}$

at lowest MW powers, three additional lines are detected at $g=2.017,2.003$ and 1.992 (resp. $\mathrm{L}_{5}$, $\mathrm{L}_{6}, \mathrm{~L}_{7}$ ). (2) at highest MW power, satellite lines (shoulders) near $\mathrm{L}_{1}$ to $\mathrm{L}_{4}$ are visible, marked by black lines. (3) the FLS becomes less and less symmetric as the MW power decreases.

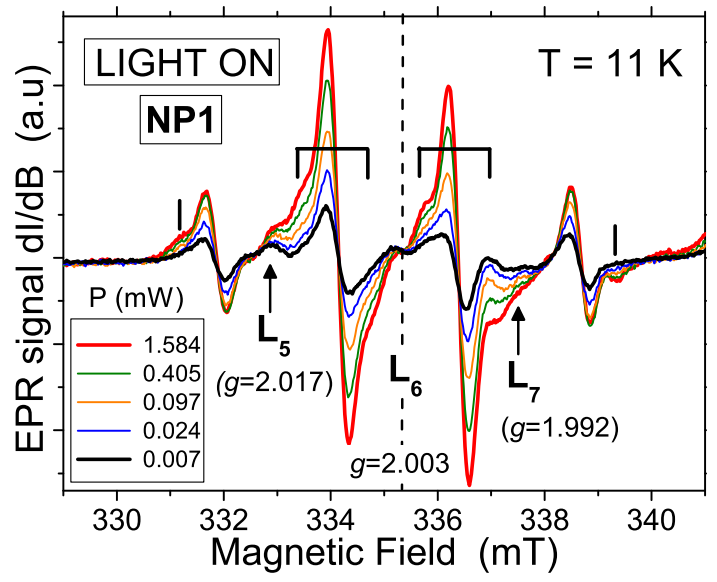

Figure 4: (color online) EPR spectra recorded on NP1 sample under white-light illumination at $T=11 \mathrm{~K}$, for five values of MW power. Black marks indicate satellite lines discussed in the text.

The spectra dependence on MW power (Fig. 4) shows that the three additional-lines intensity $\left(\mathrm{L}_{5}\right.$ to $\mathrm{L}_{7}$ ) are almost power-independent, while the FLS intensity notably decreases with power. This signs a basic difference between the dynamics of these lines and the FLS, the formers having longer relaxation time than the latter. $\mathrm{L}_{5}$ and $\mathrm{L}_{7}$ displays no evidence for a relation with the FLS, whereas $\mathrm{L}_{6}(g=2.003)$ is likely to be the original SD defect itself, which is also fully saturated at $11 \mathrm{~K}$. Indeed, in other kind of samples in which the FLS is observed under illumination (see NP2 Fig. 10 and Sec. IV), $\mathrm{L}_{5}$ is also present with different $g$-valOmerue, while $\mathrm{L}_{6}$ and FLS have the same position. It may be assumed that the photo-induced FLS is related to the SD, while being although of different nature, and that $L_{5}$ and $L_{7}$ arise from versatile excited defects, depending on growth method and conditions.

The satellite lines of the FLS appear only at highest powers, and each of them is located at a distance value $\sim \Delta / 4$ from the nearest FLS line. Interestingly, $\mathrm{L}_{2}$ and $\mathrm{L}_{3}$ appear to be accompanied by two of these satellite lines, while $\mathrm{L}_{1}$ and $\mathrm{L}_{4}$ by only one. These satellites are likely to arise from multiphotons absorption, forbidden transitions, or low symmetry fields. The increase of their intensity is mainly responsible for the line-shape deformation at high power.

Regarding the FLS itself, its intensity varies notably with MW power variation, while preserving a constant $\Delta$ spacing and gravity center, and it becomes more and more asymmetric as the MW-power decreases. However, it can be seen on Fig. 5 that intensity of lines $\mathrm{L}_{1}$ and $\mathrm{L}_{4}$ do not vary anymore from $P=0.405 \mathrm{~mW}$ to $P=1.584 \mathrm{~mW}$, contrarily to the center lines $\mathrm{L}_{2}$ and $\mathrm{L}_{3}$. This indicates a slight difference in the dynamics of these transitions, which will be quantified in the following and discussed in Sec. IV. The FLS line widths are not affected by the MW power variation.

We now turn to the examination at higher temperature $(85 \mathrm{~K})$ of the FLS in order to observe fully unsaturated resonance, for it is the only case which can be treated by simulations.

EPR spectra recorded on NP1 sample under illumination at $T=85 \mathrm{~K}$ are shown in Fig. 5, for different MW powers. The widths of the $\mathrm{L}_{5}$ and $\mathrm{L}_{6}$ lines are weaker than at $T=11 \mathrm{~K}$, certainly due to partial desaturation, which allows for an almost complete resolution of them. On the contrary, the $\mathrm{L}_{7}$ line is still not resolved. Line widths of FLS are reduced from $0.4 \mathrm{mT}$ to $0.3 \mathrm{mT}$. The satellite lines are relatively much weaker, still only appearing for the highest MW powers.

The interesting feature of the spectra plotted in figure 5 is the observation of three completly different EPR-line dynamics. First, $\mathrm{L}_{1}$ to $\mathrm{L}_{4}$ lines do not reach a total saturation regime, since their intensity always increases with increasing MW power. Beside, the $\mathrm{L}_{5}$ line reaches a total saturation regime for MW power of $0.79 \mathrm{~mW}$, while $\mathrm{L}_{6}$ and $\mathrm{L}_{7}$ lines appears to be always fully saturated, at any MW power. This is a srong indication that all these line groups arise from differents photo-excited paramagnetic centers. 


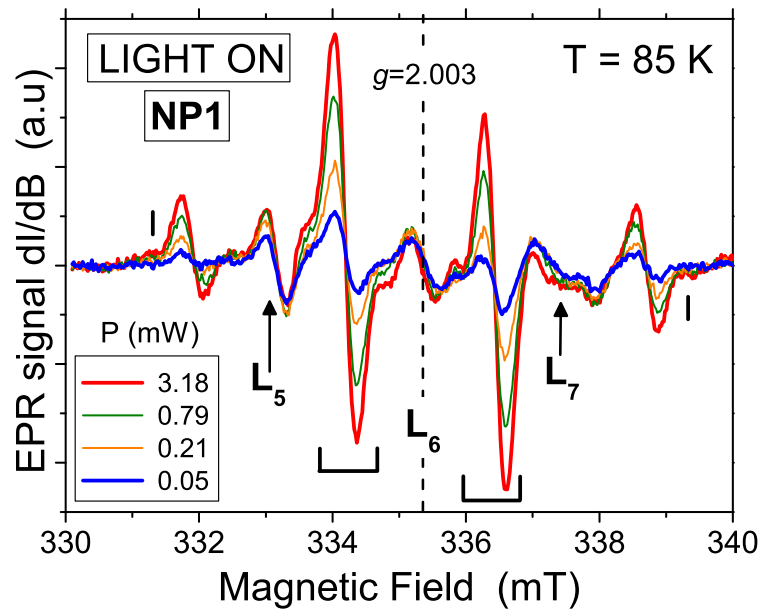

Figure 5: (color online) EPR spectra under illumination recorded on NP1 sample at $85 \mathrm{~K}$ (colored, bottom) for different MW powers, and at $9 \mathrm{~K}$ (black, top) for $P=0.21 \mathrm{~mW}$. Magnetic-field modulation is $2 \mathrm{G}$. Satellite line are indicated by black marks.

A key physical information to be extracted from these spectra is obtained from their intensity MW power dependence, which gives insight into the dynamics of each EPR line, as well as into the spin-resonance regime (saturated or unsaturated). Peak-to-peak height for lines $\mathrm{L}_{1}$ to $\mathrm{L}_{4}$ (proportional to absorbed intensities for constant peak-to-peak line width) have been plotted as functions of MW power in Fig. 6, at 11 and $85 \mathrm{~K}$. It is to recall that an EPR line is said to be not saturated if by doubling $\sqrt{P}$, the intensity is doubled. From this simple criterium, we see that at $T=85 \mathrm{~K}$, the FLS is not saturated only for incident MW power as weak as $P=0.05 \mathrm{~mW}$. At $T=11 \mathrm{~K}$, whatever the weakness of the incident MW power is, the FLS structure is always saturated: the required power for a total unsaturated regime of the FLS is so low that the lines cannot be detected anymore in our experimental conditions.

More quantitative information about EPR dynamics is obtained by the fitting of the saturation curves with an appropriate function including physical parameters. The simplest modeling of the saturation phenomena is derived from the isotropic Bloch equations, including longitudinal and transverse relaxation times $T_{1}$ and $T_{2}$. It results in the following power dependence of the EPR intensity ${ }^{46}$ :

$$
I(P)=\frac{K \cdot \sqrt{P}}{1+A \cdot P}
$$

The parameter $A$ is proportional to the product of the relaxation times $T_{1}$ (spin-lattice) and $T_{2}$ (spin-spin). It determines the maximum of the

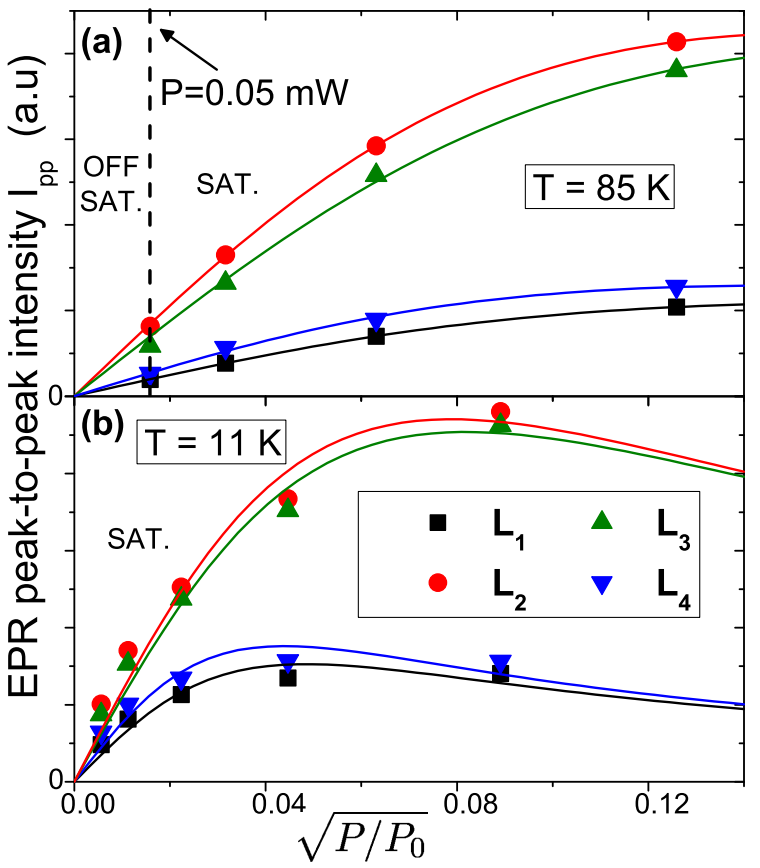

Figure 6: (color online) Experimental saturation curves of the FLS (scatters) recorded on NP1 sample at $T=$ $85 \mathrm{~K}$ (a) and $T=11 \mathrm{~K}$ (b), and fit from Eq. (1) (solid lines).

saturation curve. The parameter $K$ contains the square of the transition probability, the Boltzmann factor, and the $T_{2}$ time. The fitting of EPR intensities vs $\sqrt{P}$ is displayed in Fig. 6 , which show an overall good agrement. At $T=85 \mathrm{~K}$, parameter $A$ is found to be almost identical for the four considered lines. However, at $T=11 \mathrm{~K}$, saturation curves clearly show that $\mathrm{L}_{1}$ and $\mathrm{L}_{4}$ reach maximum at MW power substantially weaker than $\mathrm{L}_{2}$ and $\mathrm{L}_{3}$. The fitting allows to determine that $A_{1,4} / A_{2,3} \simeq 3$. By assuming that $T_{2}$ is almost the same for all these lines, it is finally deduced that the spin-lattice relaxation time $T_{1}$ is notably longer for extreme lines of the FLS $\left(T_{1}^{1,4} / T_{1}^{2,3} \simeq 3\right)$. This fact will be of importance in the following, when searching for the appropriate spin Hamiltonian to fit the FLS.

Finally, we note that after light-irradiation removal the FLS remains detectable for several hour at $85 \mathrm{~K}$, which will be discussed in Sec. IV.

\section{Simulations}

In order to gain insight into the origin of the photo-induced FLS, spectra simulations have been performed, based on assumed spin Hamiltonian and spin state. It is found that two radically different spin systems can give account for the observed FLS. This latter can arise either (1), from superhyperfine coupling of one electronic $1 / 2$ 
spin with 3 adjacent protons, or (2), from a $S=2$ spin under weak axial anisotropy.

In the following, we present arguments which make the first scenario very unlikely to exist. Then, we show that the second one can give well account for the experimental spectra.

In fact, a complex made of one $1 / 2$ spin (hole or electron) surrounded by 3 protons can exist in $\mathrm{ZnO}^{47,48}$. These protons can be provided by the ubiquitous unintentional hydrogen-doping, and the $1 / 2$ spin can be carried by a point defect. However, in such a case, signals arising from simpler complexes (involving only 1 or 2 protons) must then occur with higher probabilities, which are indeed not detected. In addition, due to the identical $\Delta$ spacing between lines, the superhyperfine coupling constant would have to be exactly the same for all protons, this constant $(A=66 \mathrm{MHz})$ being moreover very intense for such a distant interaction $(A=1.4 \mathrm{MHz}$ for donor spin coupling with adjacent hydrogen in $\mathrm{ZnO}^{49}$ ). It is also to be noted that the FLS has been observed in other kind of hydrothermally grown samples with exactly the same $\Delta$ spacing: this indicates a stable and reproducible property, poorly compatible with random distribution of unintentional hydrogen impurities.

Finally, the most important argument against this first scenario, and in favor of the second one, is provided by the saturation curves of the FLS (Fig. 6). These show that at $T=11 \mathrm{~K}$ the extreme lines $\mathrm{L}_{1}$ and $\mathrm{L}_{4}$ have not the same dynamics than that of the center lines, $\mathrm{L}_{2}$ and $\mathrm{L}_{3}$. This has been quantitatively demonstrated by the fits of Fig. 6, leading to the ratio of spin-lattice relaxation times $T_{1}^{1,4} / T_{1}^{2,3} \simeq 3$. In the case of hyperfine couplings, however, all of the four lines would arise from $|-1 / 2\rangle \leftrightarrow|+1 / 2\rangle$ electronic-state transitions, and must then have the same spin-lattice coupling, thus saturating at the same MW power.

On the contrary, if the FLS arise from a $S=2$ spin under weak axial anisotropy, this difference in dynamics is expected because, as shown in Fig. 7-b, the extreme lines $\mathrm{L}_{1}$ and $\mathrm{L}_{4}$ arise from transitions $| \pm 2\rangle \leftrightarrow| \pm 1\rangle$, while the center lines $\mathrm{L}_{2}$ and $\mathrm{L}_{3}$ do from transitions $| \pm 1\rangle \leftrightarrow|0\rangle$. Different electronic spin states having different coupling with lattice's phonons, the above-mentioned transition must have different dynamics, consistently with our observations.

In the following, based on the above-mentioned arguments, we focus on the $S=2$ scenario and present the simulated spectra based on the spin Hamiltonian written in Figure 7-b. This latter contains the Zeeman interaction with the static magnetic field $\mathbf{B}$, and an axial anisotropy term characterized by the $D$ parameter $\left(D<g \mu_{B} B\right)$.

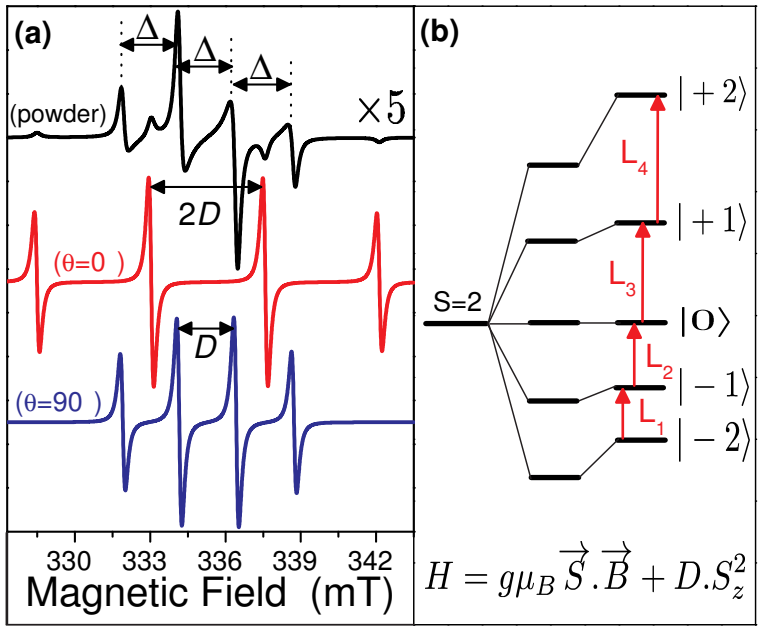

Figure 7: (color online) (a) Simulated EPR spectra for $S=2$ spin with axial anisotropy $D$ for powder, $B \| z$ $\left(\theta=0^{\circ}\right)$ and $B \perp z\left(\theta=90^{\circ}\right)$. (b) Corresponding Hamiltonian and levels scheme.

For $\theta=90^{\circ}$ orientation, the spacing between lines simply equals $D$, in magnetic field units. The simulated powder spectrum is strongly asymmetric, and is dominated by the most occurring orientation, that is $\theta=90^{\circ}$. Then, the experimental $\Delta$ spacing is a direct measure of the $D$ parameter.

Regarding simulations, it must be kept in mind that only unsaturated resonance of one-photon transitions can be accounted for by the current EPR software as EASYSPIN ${ }^{50}$ used here. Rigorously, the spectra to be simulated must be restricted to those recorded in unsaturated conditions, which is only the case at $T=85 \mathrm{~K}$ and $P=0.05 \mathrm{~mW}$ (see Fig. 6). This experimental spectrum displays the most asymmetric FLS, that well corresponds to the powder spectra of $S=2$ with axial anisotropy (Fig. 7-a), and no satellite lines around the FLS.

In order to reproduce this unsaturated experimental spectrum, the powder spectra of $S=2$ with axial anisotropy (Fig. 7-a) is used, involving only one energetic parameter $(D)$. Also the three lines $L_{5}$ to $L_{7}$ evoked before (Sec. IIIB) are added, plus another one (denoted $\mathrm{L}_{8}$ ) which appears necessary to obtain an overall good agreement. The resulting simulation, presented in figure 8 , appears very satisfactory. The axial anisotropy parameter is found to be $|D|=66 \mathrm{MHz}$ $\left(=22 \times 10^{-4} \mathrm{~cm}^{-1}\right)$, which corresponds to a very weak axial anisotropy. Its sign, which depends on the in-plane or out-of-plane character cannot be determined in our experimental conditions (too high temperature).

The number of $1 / 2$ spins used in this simulation (4) may appear as too important for drawing 


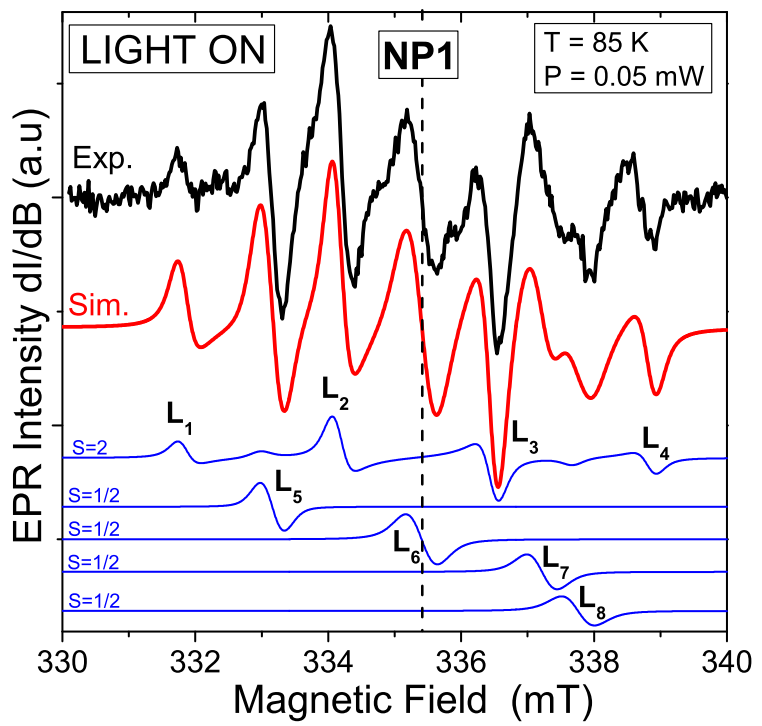

Figure 8: (color online) Simulated (red) and experimental (black) EPR spectra recorded on NP1 at $85 \mathrm{~K}$ in unsaturated conditions $(P=0.05 \mathrm{~mW})$ under illumination. Various contributions to the simulation are display (blue), all divided by a factor 3 .

conclusion about the FLS. However, these additional $1 / 2$ spins are clearly not directly related to the $\mathrm{FLS}^{59}$, so that their introduction in the simulation does not prevent to conclude about the actual presence of a $S=2$ local electronic spin.

The unsaturated spectrum being well reproduced by simulation, the saturated case is now examined in order to understand the difference between both. Apart of the satellite lines apparition briefly discussed in Sec. III B (not taken into account in simulation), the main characteristic of this experimental saturated spectrum is the almost symmetric shape of the FLS, which is not compatible with a simple anisotropic $S=2$ powder (see Fig. 7-a). The explanation can be that powder-spectrum simulation does not explicitly includes anisotropic line widths ${ }^{60}$, while it is likely to actually arise in $\mathrm{ZnO}$ saturated EPR.

Surprisingly, it can be noticed that the saturated EPR spectrum tends to look alike the $B \perp z$ simulation (Fig. 7 -a, $\theta=90^{\circ}$ ). This can be understood if the line width along the other direction $\left(\theta=0^{\circ}\right)$ becomes more and more important as the MW power is increased, so that its apparent height decrease. As a consequence, the powder spectrum is disturbed and tends to the oriented $\theta=90^{\circ}$ direction as the saturation is reached. Figure 9 presents the comparison between the saturated experimental spectrum at $85 \mathrm{~K}$ and the simulation. This latter include the $S=2$ powder spectrum, the four additional lines $\mathrm{L}_{5}$ to $\mathrm{L}_{8}$, but also a contribution from the $\theta=90^{\circ}$

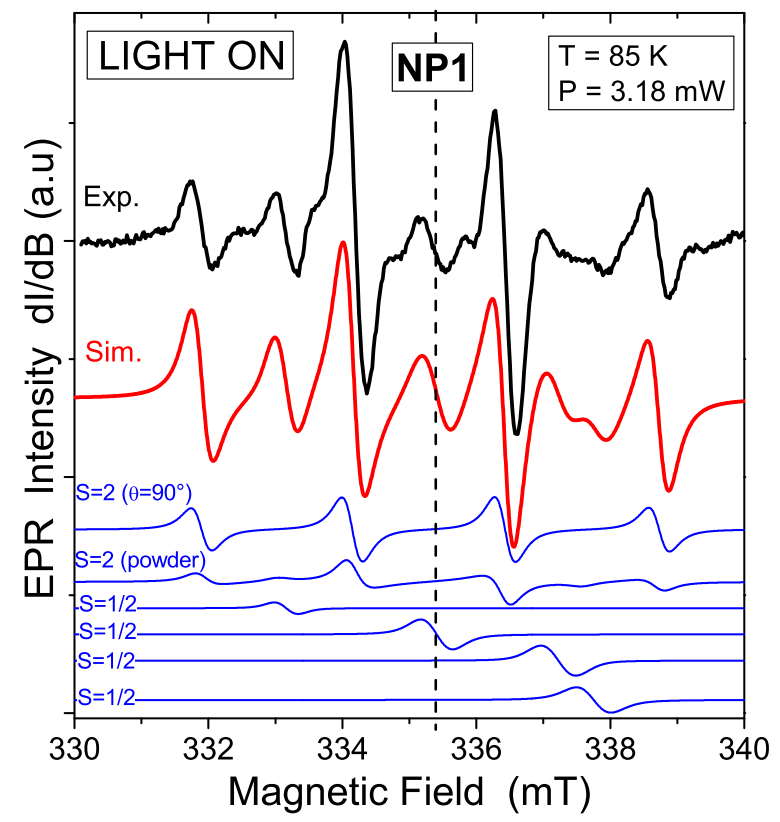

Figure 9: (color online) Simulated (red) and experimental (black) EPR spectra recorded on NP1 at 85 $\mathrm{K}$ in saturated conditions $(P=3.18 \mathrm{~mW})$ under illumination. Various contributions to the simulation are display (blue), all divided by a factor 3 .

orientated $S=2$ simulation. We may consider this last contribution as an effective way of taking into account a strong anisotropy of the EPR line width, arising in saturated cases.

In the following, it is proven that the photoinduced FLS is reproducible in other kind of dried hydrothermally-grown sample, with different size and morphology (NP2 sample). In figure 10, the EPR spectrum recorded on NP2 under white-light illumination is shown, together with its corresponding simulated EPR spectrum.

First, the photo-induced FLS is also observed, with exactly the same $\Delta$ spacing $(2.25 \mathrm{mT})$ and almost the same gravity center $(g=2.004$ vs $g=2.003$ in NP1). This points to the great stability of the paramagnetic center responsible for this EPR signal. Interestingly, the $\mathrm{L}_{7}$ and $\mathrm{L}_{8}$ lines observed in NP1 are not detected in NP2, while $\mathrm{L}_{5}$ is now positioned at a different $g$-value. This support the assumption that although all these lines are photo-induced by white light, they arise from independent paramagnetic centers and can exist independently from each other. The $\mathrm{L}_{6}$ line is still present at the gravity center of the FLS, but with greater relative intensity. Indeed, in this NP2 sample, the FLS is much weaker than in NP2 and high MW power is required in order to obtain acceptable signal-to-noise ratio. The presented experimental spectrum of figure 10 is thus recorded in saturated conditions $(P=6.32 \mathrm{~mW})$. 


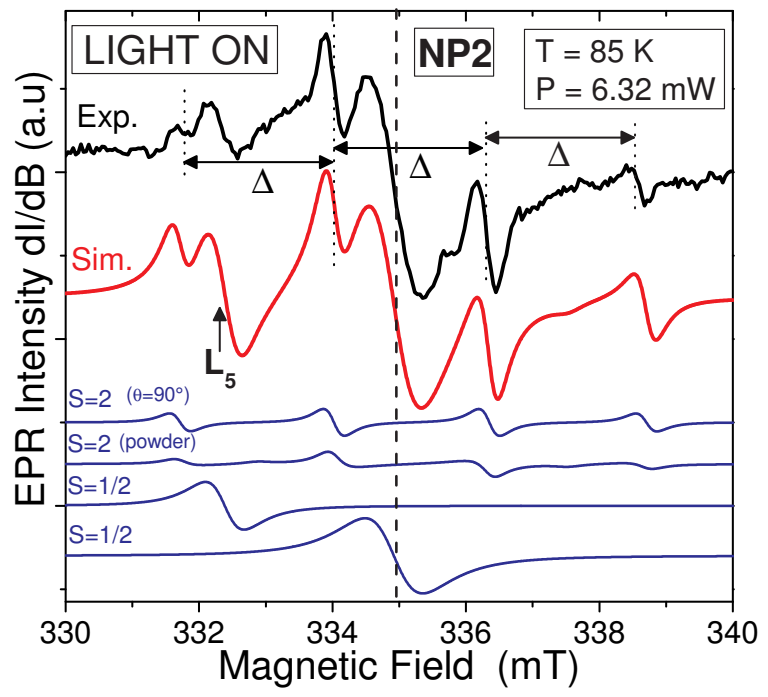

Figure 10: (color online) Experimental and simulated spectra recorded at $T=85 \mathrm{~K}$ and $P=6.32 \mathrm{~mW}$ on the S2 sample. All simulation's contributions, with their respective weight, are divided by 3 .

Regarding the simulation, and consistently with the above-presented results, such a saturated case needs the contribution of the $\theta=90^{\circ}$ orientated $S=2$ spin, for giving account of the symmetric shape of the FLS. The resulting simulation can be viewed as satisfactory.

It is then concluded that there actually exist a photo-excited $S=2$ localized magnetic state in $\mathrm{ZnO}$ NPs, together but independently of other $S=1 / 2$ photo-induced paramagnetic centers. In the following, the possible origins for this high-spin state are discussed.

\section{DISCUSSION}

Regarding the existance of the $S=2$ spin, it must be noticed that such an even high spin state is quite rarely observed in matter, where strong local anisotropy often prevent the related EPR transitions to occur $\left(\Delta m_{S}= \pm 1\right.$ required $)$. The present observation of $S=2$ spin with very weak axial anisotropy $(|D|=66 \mathrm{MHz})$ thus excludes the possibility for a transition metal ion origin, such as the ${ }^{5} \mathrm{D}$ ground multiplet of $\mathrm{Fe}^{2+}$ ion, which would be expected to have larger axial anisotropy parameter $D$. For comparison, $D=-710 \mathrm{MHz}$ in $\mathrm{ZnO}: \mathrm{Mn}^{2+}(S=5 / 2)^{51}$, and $D=+82743 \mathrm{MHz}$ in $\mathrm{ZnO}: \mathrm{Co}^{2+}(S=3 / 2)^{52}$.

Focusing on an intrinsic-defect origin for the observed photo-induced FLS, we recall that the assumed $S=2$ spin-state must be viewed as effective, possibly made of orbital and spin magnetic momentum.
Without orbital contribution, the existence of a $S=2$ spin requires the local coupling of at least four $1 / 2$ spins (bear by electrons or holes). Such a situation can exist if two neutral vacancies defects in triplet state $(S=1)$ are ferromagnetically coupled. Indeed, it has been theoretically demonstrated that neutral zinc vacancy (two localized holes) has triplet ground state, while neutral oxygen vacancy (two localized electrons) has triplet excited state, lying at about $2-3 \mathrm{eV}$ above its singlet ground state ${ }^{53}$. It is thus conceivable that, if two of these vacancies lay closed together enough, a photon absorption may causes this complex to transit to an $S=2$ spin state. However, such a spin-pair would be expected to have different coupling constant, according to the relative position of each spin within the $\mathrm{ZnO}$ matrix, leading to a distribution in the axial anisotropy parameter $D$.

On the contrary, the observed $S=2$ spin appears to arise from a unique local center, with stable and identical properties among different samples. A plausible explanation for such an intrinsic high-spin center created by light irradiation in $\mathrm{ZnO}$ can be related to excitons, which are known to be possibly bounded to defect ${ }^{23,54-56}$. At least three different coupling schemes involving excitons can be thought about. (i) a tripletstate $(S=1)$ exciton can be ferromagnetically bounded to a triplet-state defect (neutral $\mathrm{V}_{Z n}$ or $\mathrm{V}_{0}$ discussed above), resulting in the observed quintet state. (ii) Two triplet excitons can be coupled together, forming a so-called high-spin state bi-exciton 57,58 , potentially localized at the surface of NPs. (iii) In cubic crystal of local symmetry $\mathrm{T}_{d}$, single exciton can exist in a quintet state $(S \equiv J=2$ effective spin), if the hole in the $J=3 / 2\left(\Gamma_{8}\right)$ valence band and the electron in the $J=1 / 2\left(\Gamma_{6}\right)$ conduction band are coupled by ferromagnetic exchange interaction. In wurtzite crystal (local symmetry $\mathrm{C}_{6 v}$ ), the $J=3 / 2$ valence band is moreover split into $\pm 3 / 2$ ( $\Gamma_{9}$, heavy hole) and $\pm 1 / 2\left(\Gamma_{7}\right.$, light hole) band ${ }^{56}$. Again, in case of ferromagnetic exchange coupling between hole and electron, this situation can also give rise to an effective $S \equiv J=2$ spin, but with weak axial anisotropy, as observed here. This last scenario is probably the most relevant, because it involved only one simple excitation.

In any cases, even that of a FLS extrinsic origin, high local electric fields at the $\mathrm{ZnO}$ NPs surface certainly play a major role in the formation and localization of such excitonic high spin.

Among the experimental facts which can confirm or disconfirm the proposed scenarios, there may be the apparition of the satellite lines at high MW power, which are not taken into account by our simulations. These are likely to arise from multiphotons absorption, but their position 
are difficult to explain, in particular the single satellite of the extreme lines $\mathrm{L}_{1}$ and $\mathrm{L}_{4}$, moreover outside of the FLS. Forbidden transitions or low symmetry Hamiltonian components mays also have to be considered to explain the satellite lines. In parallel, the study of the FLS signal decay after light removal gives a key information for its understanding as well as for its application. So far, it has been observed that the FLS has a very long life time, losing about $10 \%$ of its intensity value two hours after the light removal, at $85 \mathrm{~K}$. The only mean of speed up this decay is to heat the sample up to room temperature. This unusual very long life-time may be interpreted as the result of the high-spin state forbidden recombination, in a sort of "magnetic phosphorescence". This point is particularly interesting for potential application.

We now point to the intriguing fact that such an interesting photo-induced EPR signal has not been reported before to our knowledge. In particular, in their work onto EPR of ZnO NPs, Erdem al. have illuminated their samples with red $(671 \mathrm{~nm})$, green (532 $\mathrm{nm}$ ) and blue (445 nm) laser lights, without seeing any photo-induced FLS ${ }^{41}$. The reason is certainly that these excitation energies are simply too low to give rise to the FLS. Indeed, we have performed the same photo-EPR experiment with violet laser light $(405 \mathrm{~nm})$, and observed the FLS just as with white light illumination. From these preliminary experiments, it can be assumed that this minimal energy lay between $2.79 \mathrm{eV}$ (445 nm) and $3.06 \mathrm{eV}$ (405 nm), which would be compatible with a highly bound exciton.

A complete spectral study (up to UV energies) of this photo-induced FLS would be an important source of information for determining its origin. For example, if the FLS $\left(\mathrm{L}_{1}\right.$ to $\left.\mathrm{L}_{4}\right)$ and the additional lines $\left(\mathrm{L}_{5}\right.$ to $\left.\mathrm{L}_{8}\right)$ are really independent, their minimal excitation energy must certainly be different. Then, illuminating the sample with the lowest of these energies excitation would permit to separate the lines.

Nevertheless, excitation energy is not the only crucial parameter for observing the photo-induced FLS. In fact, in some other samples of hydrothermally grown $\mathrm{ZnO} \mathrm{NPs,} \mathrm{the} \mathrm{photo-induced} \mathrm{FLS} \mathrm{is}$ not observed. Morphology, solvent, drying and storage conditions, must have to be considered in a systematic study in order to find the exact con- ditions for favoring the FLS presence. However, we have observed it in several different samples, grown in two different labs, with exactly the same $\Delta=2.25 \mathrm{mT}$ spacing between lines. We thus believe it to be a reproducible intrinsic property of $\mathrm{ZnO}$ NPs.

In any cases, the control and the manipulation of such a high-spin state carried by a single NP open the way to promising possibility in terms of information storage or processing. Since a particular maximal wavelength must be able to switch on the high-spin state, it is very likely that some other wavelength would be able to switch it off. Thus, a light-writable and -erasable magnetic memory would be realized, readable by EPR. In such a context, the observed long life-time of the photo-induced FLS would be an advantage to preserve information with minimal refreshing. The presented results could path the way for reversibly encoding magnetic information within a stable single crystalline NP.

\section{CONCLUSION}

In this paper, we have reported on a new EPR signal, the FLS, reversibly induced by white-light irradiation in ZnO NPs. Power-dependence, temperature studies and spectra simulations show that this signal is very likely to arise from a localized high-spin state $S=2$ with weak axial anisotropy. At $T=85 \mathrm{~K}$, this spin state can last several hours after the light-irradiation removal, certainly due to highly spin-forbidden recombination process. The intrinsic character of this light-induced EPR signal is suggested, most probably related to boundexciton physics. Other photo-induced EPR lines without structure and with different spin-lattice dynamics are detected, not apparently linked to the FLS.

\section{ACKNOWLEDGEMENT}

The authors acknowledge Prof. Bruno Guigliarelli for useful discussion and Clément Sébastio for help in the optical installation.
1 Özgür U, Hofstetter D and Morkoç H 2010 Proc. IEEE, 98, 1255

${ }^{2}$ Wang Z L 2008 ACS Nano 2 1987-1992

3 Kundu S and Nithiyanantham U 2014 Eng. Chem. Res. 5313667

4 Roth P and Williums D F 1981 J. Appl. Phys. 52
6685

${ }^{5}$ Suchea M, Christoulakis S, Moschovis K, Katsarakis N and Kiriakidis G 2006 Thin Solid Films 515551

6 Ambia M G, Islam M N and Obaidul Hakim M 1992 Journal of Materials Science 27195169

7 Zhu L and Zeng W 2017 Sensors and Actuators A: 
Physical 267242

8 Bernardini S, Benchekroun M H, Fiorido T, Aguir K, Bendahan M, Dkhil S B, Gaceur M, Ackermann J, Margeat O and Videlot-Ackermann C 2017 MDPI Proceedings 1 (4) 423

9 Dey A 2018 Materials Science and Engineering: B 229206

10 Echresh A, Chey C O, Shoushtari M Z, Nur O and Willander M 2014 J. Appl. Phys. 116193104

11 Alnoor H, Pozina G, Khranovskyy V, Liu X, Iandolo D, Willander M and Nur O 2016 J. Appl. Phys. 119 165702

12 Laiho R, Poloskin D S, Stepanov Y P, Vlasenko M $\mathrm{P}$ and Zakhvalinskii V S 2009 J. Appl. Phys. 106 013712

13 Bao J, Shalish I, Su Z, Gurwitz R, Capasso F, Wang X and Ren Z 2011 Nanoscale Res. Lett. 6404

14 Harbour J R, Chow V and Bolton J R 1974 Can. J. Chem. 523549

15 Lipovsky A, Tzitrinovich Z, Friedmann H, Applerot G, Gedanken A and Lubart R 2009 J. Phys. Chem. C 11315997

${ }^{16}$ Kayaci F, Vempati S, Donmez I, Biyikli N and Uyar T 2014 Nanoscale 610224

17 Tang Y, Zhou H, Zhang K, Ding J, Fan T and Zhang D 2015 Chem. Eng. J. 262260

18 Butkhuzi T V, Chelidze T G, Georgobiani A N, Jashiashvili D L, Khulordava T G and Tsekvava B E 1998 Phys. Rev. B 5810692

19 Rodnyi P A and Khodyuk I V 2011 Opt. Spectrosc. 111776

${ }^{20}$ Vanheusden K, Seager C H, Warren W L, Tallant D R and Voigt J A 1996 Appl. Phys. Lett. 68403

${ }^{21}$ Bera A and Basak D 2009 Appl. Phys. Lett. 94 163119

22 Titov V V, Lisachenko A A, Akopyan I Kh, Labzowskaya M E and Novikov B V 2018 J. Lumin. 195153

23 Meng X, Zhou Y, Zeng X, Chen X and Chu Y 2018 J. Lumin. 195201

${ }^{24}$ Lv J, Li C and Chai Z 2019 J. Lumin. 208225

25 Kasai P H 1963 Phys. Rev. 1303989

${ }^{26}$ Galland D and Herve A 1970 Phys. Lett. A 331

27 Galland D and Herve A 1974 Solid State Commun. 14953

28 Pöppl A and Völkel G 1989 Phys. Stat. Sol. (a) 115 247

29 Carlos W E, Glaser E R and Look D C 2001 Physica B 308-310 976

30 Vlasenko L S 2009 Physica B 4044774

31 Vlasenko L S 2010 Appl. Magn. Reson. 39103

32 Jakes P J and Erdem E 2011 Phys. Stat. Sol. RRL 5256

33 Kaftelen H, Ocakoglu K, Thomann R, Tu S, Weber S and Erdem E 2012 Phys. Rev. B 86014113

34 Ruf T, Repp S, Urban J, Thomann R and Erdem E 2016 J. Nanopart. Res. 18109

${ }^{35}$ Lambe J, Baker J and Kikuchi C 1959 Phys. Rev. Lett. 36270

36 Holton W C, Schneider J and Estle T L 1964 Phys.
Rev. 133 6A A1638

37 Pöppl A and Völkel G 1990 Phys. Stat. Sol. (a) 121 195

38 Pöppl A and Völkel G 1991 Phys. Stat. Sol. (a) 125 571

39 Nikitenko V A 1992 J. Appl. Spectrosc. 57783

${ }^{40}$ Wang X J, Vlasenko L S, Pearton S J, Chen W M and Buyanova I A 2009 J. Phys. D: Appl. Phys. 42 175411

41 Erdem E 2014 J. Alloy. Compd. 60534

42 Diallo A K, Gaceur M, Berton N, Margeat O, Ackermann J and Videlot-Ackermann C 2013 Superlattices Microstruct. 58144

43 Adam R E, Pozina G, Willander M and Nur O 2018 Nanostructures and Photonics 3211

44 Savoyant A, Alnoor H, Bertaina S, Nur O and Willander M 2017 Nanotechnology 28035705

45 Savoyant A, Alnoor H, Pilone O, Nur O and Willander M 2017 Nanotechnology 28285705

46 Weil J A and Bolton R, Electron Paramagnetic Resonance, Wiley-Interscience p. 314.

47 Van de Walle C G 2000 Phys. Rev. Lett. 8551012

${ }^{48}$ Karazhanov S Zh, Marstein E S and Holt A 2009 J. Appl. Phys. 105033712

49 Hofmann D M, Hofstaetter A, Leiter F, Zhou H, Henecker F, Meyer B K, Orlinskii S B, Schmidt J and Baranov P G 2002 Phys. Rev. Lett. 88, 4, 045504

50 Stoll S and Schweiger A 2006 J. Magn. Reson. 178 45

51 Savoyant A, Stepanov S, Kuzian R, Deparis C, Morhain C and Grasza K 2009 Phys. Rev. B 80 115203

52 Sati P, Hayn R, Kuzian R, Régnier S, Schäfer S, Stepanov A, Morhain C, Deparis C, Laügt M, Goiran M and Golacki Z 2006 Phys. Rev. Lett. 96 017203

53 Chanier T, Opahle I, Sargolzaei M, Hayn R and Lannoo M 2008 Phys. Rev. Lett. 100026405

${ }^{54}$ Thomas D G and Hopfield J J 1961 Phys. Rev. Lett. $\mathbf{7}, 8,316$

55 Reynolds D C, Look D C, Jogai B, Litton C W, Collins T C, Harsch W and Cantwell G 1998 Phys. Rev. B $5 \mathbf{7} 1912151$

56 Meyer B K, Alves H, Hofmann D M, Kriegseis W, Forster D, Bertram F, Christen J, Hoffmann A, Strasburg M, Dworzak M, Haboeck U and Rodina A V 2004 Phys. Stat. Sol. (b) 2412231

57 Poem E, Kodriano Y, Tradonsky C, Lindner N H, Gerardot B D, Petroff P M and Gershoni D 2011 Nature Physics 612

58 Weiss L R, Bayliss S L, Kraffert F, Thorley K J, Anthony J E, Bittl R, Friend R H, Rao A, Greenham N C and Behrends J 2016 Nature Physics 13, 176

59 Dynamics are completly different and, moreover, in NP2, the same FLS appears without $\mathrm{L}_{7}$ and $\mathrm{L}_{8}$, and with $\mathrm{L}_{5}$ at a different position

60 EASYSPIN only take into account effective line width anisotropy due to unresolved hyperfine structure via $\mathrm{H}$-strain. 